\title{
VIDEO HALFTONING PRESERVING TEMPORAL CONSISTENCY
}

\author{
Chao-Yong Hsu, ${ }^{1,2}$ Chun-Shien Lu, ${ }^{2, *}$ and Soo-Chang Pei ${ }^{1}$ \\ ${ }^{1}$ Graduate Institute of Communication Eng., National Taiwan University, Taipei, Taiwan, ROC \\ ${ }^{2}$ Institute of Information Science, Academia Sinica, Taipei, Taiwan, ROC
}

\begin{abstract}
Video halftoning is a key technology for use in the new display device, electronic paper (e-paper). This is still a rather unexplored field. The challenging issue of video halftoning is the elimination of flicker flaw that will appear due to error diffusion in the temporal domain. In this paper, we propose a new video halftoning method, which is composed of spatial error diffusion and inter-frame reference error diffusion. In addition, since our method can efficiently reduce the flicker flaws, another advantage is that the halftone video sequence can be efficiently compressed. When compared with traditional $2 D$ and $3 D$ error diffusion techniques, experimental results show that our method can significantly reduce the average flicker rates.
\end{abstract}

\section{INTRODUCTION}

Monitor industry is actively finding new display technology to make the monitor lighter, thinner, and more portable. The electronic paper (e-paper) is exactly the advanced and emerging display technology. Compared with traditional monitors, e-paper also consumes little power. Since a famous manufacturer, e-ink, already began to sell their products in 2005, it can be envisioned in the future that most applications based on traditional paper and liquid crystal display (LCD) will be replaced by e-paper. Since digital halftone image is the only format that many electronic devices can read, the development of digital halftone image/video technique is the key to meet the requirement of e-paper. In view of this, the goal of this paper is to develop a new digital halftone video method for e-paper.

Digital halftoning $[7,10]$ refers to the physical process of converting a continuous tone images to black and white dots. Video halftoning is a technique that transfers a general video sequence into a format that can be displayed on devices with limited intensity resolutions and color palettes. In the literature, only few video halftoning techniques were discussed. A three-dimensional (3D) error diffusion algorithm that is used to mitigate the "flicker" flaw was proposed in [4]. Gotsman [3] applied an iterative image halftoning

*Corresponding Author: Dr. C. S. Lu (1cs@iis.sinica.edu.tw) algorithm to deal with the problem when temporal flickers exist between video frames. This algorithm achieves visual results better than those obtained from classic halftoning algorithms at the expense of increasing computational load. In [1], Atkins et al. investigated the display of color image sequences using a model-based approach for multilevel error diffusion. Their algorithm achieves an improvement in image quality over that yielded by frame-independent quantization when the frame rate is sufficiently high to support temporal average by the human visual system. In [5], a direct-binary-search algorithm was applied to 3-D error diffusion. In [9], Sun presented a motion adaptive gain controlbased 3-D error diffusion method to enhance the temporal consistency of the visual patterns by minimizing the flicker flaws.

However, we find that flicker flaws still are not efficiently reduced by means of the existing methods. In this paper, we will focus on developing a new digital halftoning video technique to solve the problem of flicker flaws for use in e-paper. Furthermore, since our method can efficiently reduce the flicker flaws, another advantage is that the halftone video sequence can be efficiently compressed. Extensive experimental results show that our method can significantly reduce the flicker rates without raising other side effects.

\section{FLICKER FLAWS IN VIDEO HALFTONING}

A general video halftoning method consists of spatial error diffusion and temporal error diffusion, both of which create the flicker phenomenon. Flicker means the change of halftone values (either from black to while or from white to black) that will be easily perceived by human eyes. For temporal error diffusion, this procedure will cause the pixels located at the same positions of neighboring video frames to have different halftone values even the pixels have the same or similar gray values. For spatial error diffusion, since the probability that a pixel with gray level 128 halftoned to a white or black halftone value is the same, the flicker flaw will be generated when the pixel values are close to 128 . Fig. 1 shows the effect of flicker flaws. Specifically, the while dots in Fig. 1(c) indicate the changes of halftone val- 
ues, which will make human eye uncomfortable when we view Figs. 1(a) and (b) successively.

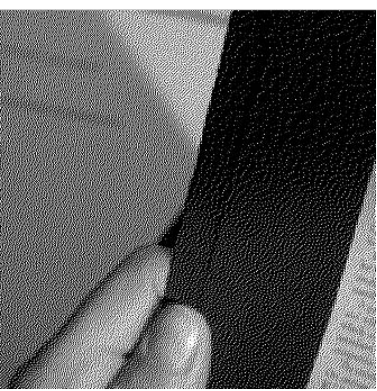

(a)

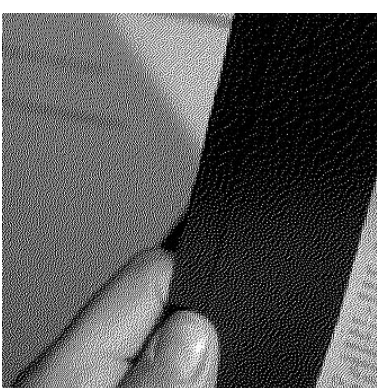

(b)

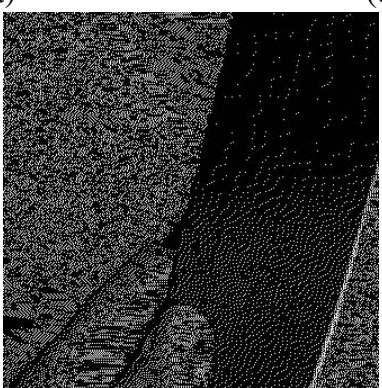

(c)

Fig. 1. Flicker flaws: (a) and (b) show the neighboring halftone video frames; (c) shows the flicker flaws, illuminated with white dots, between (a) and (b).

\section{OUR METHOD}

\subsection{New Video Halftoning based on Inter-Frame Ref- erence Error Diffusion}

In this section, we describe a new video halftoning method, which is composed of intra-frame error diffusion and interframe reference, to reduce flicker flaws in halftone video. Compared with the existing methods, our method avoids adopting 3D error diffusion. Fig. 2 shows the block diagrams of the proposed method.

As shown in Fig. 2, the first video frame, $I\left(x, y, z_{1}\right)$, is halftoned by spatial error diffusion [2] to generate the first halftone frame $H\left(x, y, z_{1}\right)$. To reduce the flicker flaws, the remaining halftone video frames are not generated by means of spatial error diffusion. On the contrary, each of them is compared with its previous frame during the subsequent halftoning process. For example, the second frame $I\left(x, y, z_{2}\right)$ is compared with the first one for inter-frame error diffusion. If the gray level difference between two pixels located at the same position is smaller than a threshold, then the halftone value of the pixel in the second frame is assigned to be the same as that in the first frame; otherwise, the halftone value is determined via a quantization process

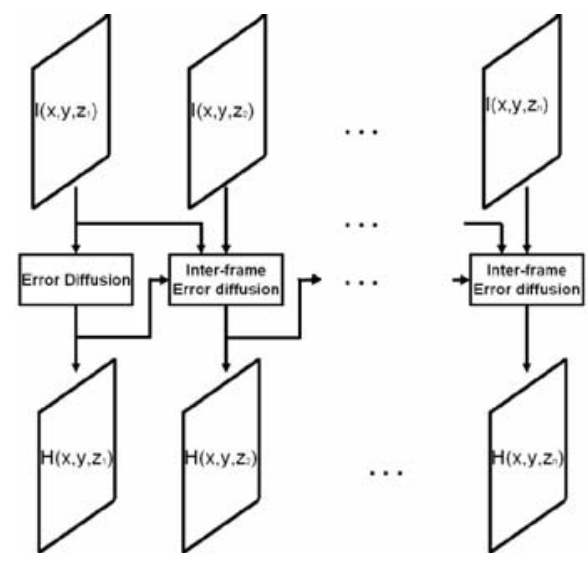

Fig. 2. Block diagrams of our method.

in error diffusion. Despite which kind of conditions occurs, the quantization errors are still needed to be diffused.

In the proposed video halftoning method, video frames are halftoned sequentially. In each frame, the pixels are processed in the order, from left to right and up to bottom.

\subsection{Spot Defects and Their Error Propagation}

In our method, inter-frame reference is used to block the propagation of flicker flaws. However, the side effect is that the quantization errors are gathered but not properly diffused. In particular, if the quantization errors are gathered at the areas where the differences between two neighboring frames are small, then the pixels with similar gray levels will be halftoned to different halftone values because the quantity of quantization errors is too large to be completely diffused. This will cause an abnormally halftoned area to be filled with white/black dots when positive/negative quantization errors are gathered. We call the result of this kind of error gathering, "spot defect." Unfortunately, the visual quality of halftone video will be damaged because spot areas will be continuously propagated to next frames. Fig. 3 shows an example of a halftone video frame with some spot defects.

In order to reduce spot defects, the quantization errors must be properly controlled, as described in the next subsection.

\subsection{Inter-Frame Reference Video Halftoning with Spot Defect Elimination}

The proposed video halftoning algorithm described in Sec. 3.1 proposes to use inter-frame reference for flicker elimination. The drawback is that spot defects and their error propagation are introduced. In order to cope with these problems, two things must be done: (i) accumulated quantization errors have to be released; (ii) error propagation has 


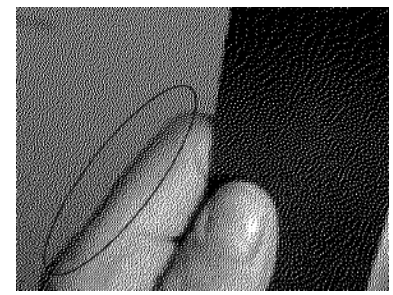

(a)

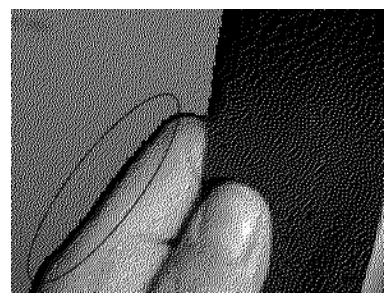

(b)
Fig. 3. Spot defect in a halftone video frame generated using inter-frame reference error diffusion: (a) original frame; (b) halftone frame with spot defect on the finger.

to be suppressed.

In order to completely release the quantization errors generated during the halftoning process, they must be controlled to not exceed the maximum value +1 or -1 . If the quantity of quantization error accumulated at a pixel exceeds 1 , then its halftone value cannot be determined using inter-frame reference. On the contrary, the halftone value must be set to the value opposite to that decided using interframe reference in order not to accumulate more quantization errors. The cost is that the flicker flaw at single points will occur. However, single flicker point will not lead to apparent visual quality degradation.

In order to endow the proposed method with the ability of suppressing error propagation, similar to video coding, the video frames are divided into many group of pictures (GOPs) for video halftoning. Each GOP consists of an I frame and a number of $P$ frames, where $P$ frames refer to I frame during the halftoning process. There may be two ways to achieve the segmentation of GOPs. The first one is similar to video shot change detection in that an abrupt change of video content will represent an I frame, i.e., a start of a new GOP. This is done in the gray-scale or color domain. The second one refers to the change of average flicker rate to determine the existence of an I frame and is adopted in this paper. In order to evaluate the performance of flicker reduction, the average flicker rate (AFR) between a pair of neighboring halftone video frames defined as

$$
\left.A F R=\sum_{y=1}^{Y} \sum_{x=1}^{X}|I(x, y, z)-I(x, y, z-1)|\right) /(X \cdot Y)
$$

is used as a metric for indicating the degree of flicker flaw. In Eq. (1), $X \times Y$ denotes the size of a video frame and $z$ denotes frame number. If $A F R(0 \leq A F R \leq 1)$ is larger than a threshold, then the $z$ frame is regarded as an I frame, which is halftoned by means of $2 \mathrm{D}$ error diffusion. Since I frame is used to refresh the halftoning process, spot defects can be blocked at the expense of increasing flicker flaws between I frame and its previous frame. As for P frames, the algorithm described in Sec. 3.1 is used to achieve video halftoning.

\subsection{Efficient Halftone Video Compression}

Since flicker flaw can be efficiently reduced by our method, the background during a series of video frames can be almost kept the same. As a result, this will lead to efficient compression of a halftone video sequence by saving a lot of bit rates used for encoding the changeable background due to the introduction of flicker flaws. In addition, it is worth noting that since the GOP structure is adopted in our video halftoning method, compression of halftone video is compatible to the current video coding standards.

\section{EXPERIMENTAL RESULTS}

In the experiments, a number of 24-bits color video sequences were used. Among them, the results obtained from the "Vassar" video [8], "Secretary" video, which was excerpted from the movie "The devil wears the Prada," and "Dolphin" video, which was captured by a hand-held camera, are reported here. These video sequences, respectively, contain small, middle, and large motion contents. The frame size ranges from $320 \times 240$ to $436 \times 1024$.

In order to evaluate the quality of halftone video by means of temporal consistency, the change of flicker rate (Eq. (1)) is a good indicator. Fig. 4 Fig. 6 show the average fliker rate of every pair of neighboring halftone frames for the "Vassar", "Secretary", and "Dolphin" video sequences, respectively. In these figures, the 3D error diffusion method [4], the 2D error diffusion method [2], and our method were used for comparison. It can be observed from these results that (i) 3D error diffusion outperforms $2 \mathrm{D}$ error diffusion in video halftoning; (ii) our method consistently obtains the lowest average flicker rates among the three methods. We also find that some AFRs of the three methods are very close for those frames with large motions. In fact, they (the peaks in the curve generated using our method) occur in I frames. Furthermore, we find that for videos with large motions more I frame will be determined (e.g., many peaks appear in Fig. 6).

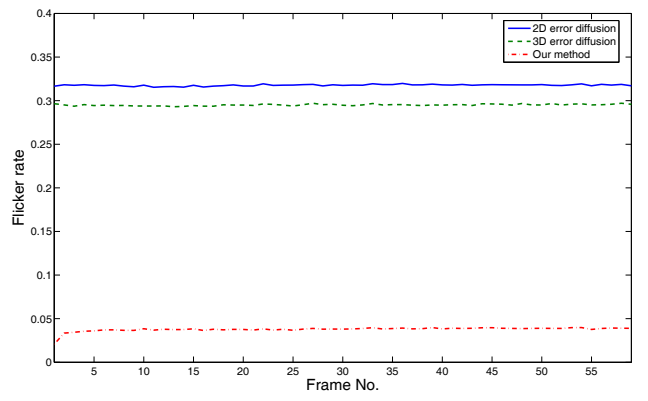

Fig. 4. Comparison of average flicker rates for the Vassar video. 


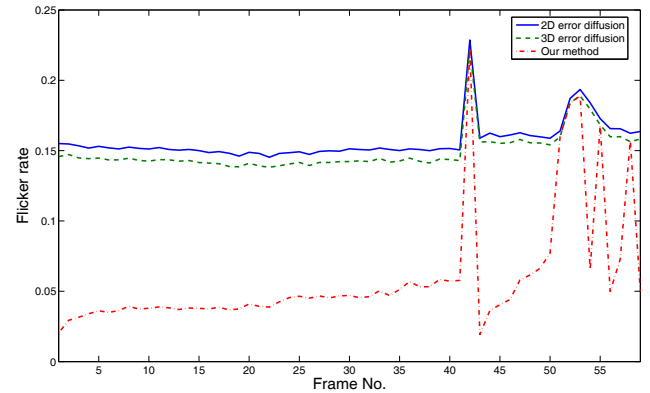

Fig. 5. Comparison of average flicker rates for the Secretary video.

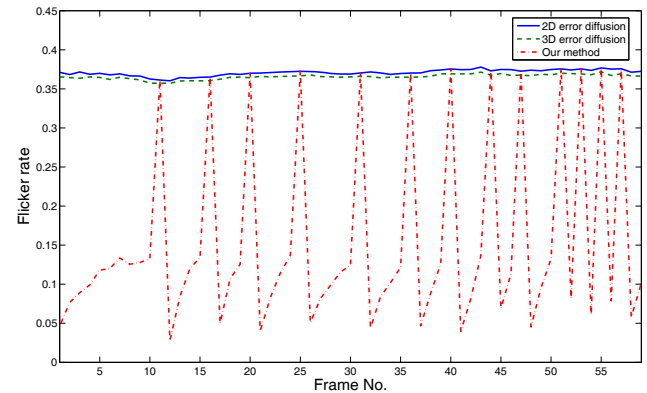

Fig. 6. Comparison of average flicker rates for the Dolphin video.

Next, we examine how spot defects can be eliminated based on our GOP-based inter-frame reference error diffusion method. Fig. 7 shows the result of spot defect elimination. Compared with Fig. 3(b), it is found that the spot defects have almost been deleted. In other words, both flicker flaws and the derived spot defects can be efficiently deleted by means of the proposed method.

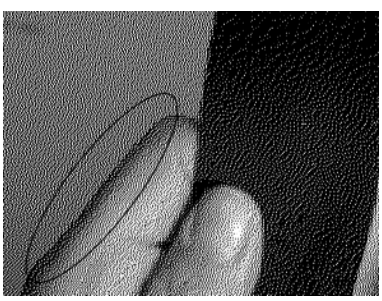

Fig. 7. Elimination of spot defects in a halftone video frame.

\section{CONCLUSION}

In view of the fact that video halftoning will play an important role in supporting the use of the emerging display device, e-paper, this paper proposes a new video halftoning method that can preserve temporal consistency between video frames. Another advantage is that efficient compression of halftone video can be achieved due to the background content does not incur flickers. Experimental results and comparisons with known methods indeed demonstrate the excellent performance of our method.

When e-paper achieves to be widely used, the incoming question is the copyright protection of digital halftone video sequences and images. In this aspect, we also noted that [6] the current digital halftone image watermarking methods are not suitable for protecting the output generated from high-resolution devices because even the lowest resolution of the commonly used printers and scanners achieve 100 dpi. Therefore, it is meaningless to embed watermarks into low-resolution halftone images. Based on the above reasons, we will also study a new digital hgalftone video watermarking method that is well suited for high-resolution output devices such as e-paper, laser printer, and scanner.

Acknowledgment: This work was supported by the National Science Council under NSC grant NSC 95-2422-H001-008.

\section{REFERENCES}

[1] C. B. Atkins, T. J. Flohr, D. P. Hilgenberg, C. A. Bouman, and J. P. Allebach, "Model-based color image sequence quantization," in Proc. SPIE/IST Conf. Human Vision, Visual Processing, and Digital Display V, vol. 2179, Feb. 1994, pp. 310-317.

[2] R. Floyd and L. Steinberg, "An adaptive algorithm for spatial grey scale," Proc. Soc. Inf. Display, vol. 17, no. 2, pp. 75-77, Mar. 1976.

[3] C. Gotsman, "Halftoning of image sequence," Vis. Comput., vol. 9, no. 5, pp. 255-266, 1993.

[4] H. Hild and M. Pins, "A 3-D error diffusion dither algorithm for half-tone animation on bitmap screens," in Stateof-the-Art in Computer Animation-Proceedings of Computer Animation, Berlin, Germany: Springer-Verlag, pp. 181-190, 1989.

[5] D. P. Hilgenberg, T. J. Flohr, C. B. Atkins, J. P. Allebach, and C.A. Bouman, "Least-squares model-based video halftoning," in Proc.SPIE/IST Conf. Human Vision, Visual Processing, and Digital Display V, vol. 2179, pp. 7-10, 1994.

[6] C. Y. Hsu and C. S. Lu, "Joint Image Halftoning and Watermarking in High-Resolution Digital Form," Proc. IEEE Int. Conf. on Multimedia and Expo, The Netherlands, 2005.

[7] H. R. Kang, Digital Color Halftoning. New York: SPIE/IEEE Press, 1999.

[8] Mitsubishi Electric Research Laboratories, "MERL multiview video sequences," ftp://ftp.merl.com/pub/avetro/mvctestseq.

[9] Z. Sun, "Video Halftoning," IEEE Trans. on Image Processing, vol. 15, no. 3, pp. 678-686, 2006.

[10] R. A. Ulichney, Digital Halftoning. Cambridge, MA: MIT Press, 1987. 